International Journal of Pure and Applied Mathematics

Volume 107 No. 2 2016, 439-447

ISSN: 1311-8080 (printed version); ISSN: 1314-3395 (on-line version)

url: http://www.ijpam.eu

doi: 10.12732/ijpam.v107i2.13

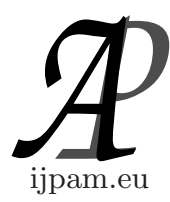

\title{
SERIES SOLUTIONS OF PREY-PREDATOR SYSTEM WITH INTRASPECIES COMPETITION USING ADOMIAN DECOMPOSITION METHOD
}

\author{
M.C. Kekana \\ Department of Mathematics and Statistics \\ Tshwane University of Technology \\ Private Bag X680, Pretoria, SOUTH AFRICA
}

\begin{abstract}
In this paper, Infinite series solutions of prey-predator system with intraspecies competition is obtained using the Adomian decomposition method. The approximated solutions are shown graphically. The results reveals that Adomian decomposition method is an altenative method of solving systems of non-linear equations. The software used in this paper is Mathematica 10.
\end{abstract}

AMS Subject Classification: 74H10, 37N25, 46N60, 65M12

Key Words: Adomian decomposition method, series solution, Adomian polynomial, preypredator system, intraspecies competition

\section{Introduction}

Analytical solution to systems of non-linear differential equations are very important in area such as biological mathematics. An interaction of increasing and decreasing in population of the species as called predator-prey models and they contains systems of non-linear differential equations.

Adomian decomposition method (ADM) is an approximate method of solving both initial valued linear and nonlinear differential equation, this method

Received: December 21, 2015

Published: April 9, 2016 (c) 2016 Academic Publications, Ltd.

url: www.acadpubl.eu 
generally split a differential equation into two parts the linear and non-linear part. The method was developed by G. Adomian, see [1, 2, 3, 4]. Intraspecific competition is a competition for which same species competiting for same resources such as water, shelter and mates to name the few.

Different results has being archived while comparing the ADM with other perturbation methods. Makinde in [9] solved the ratio-dependent prey-predator with constant effort harvesting using ADM. Edward et al (see [7]) campared ADM to Runge-Kutta on prey-predator model. Shalan et al in [10] investigated the dynamics of Holling Type IV prey-predator models with intra-specific competition. Less investigation has been done on prey-predator model that incoporate intraspecies competition.

The main aim ideas to this study is to obtain infinite series solution of prey predator model with intraspecies competition using the Adomian decomposition method.

\section{Qualitative Analysis}

Consider the following prey-predator model with Intraspicies competitions

$$
\begin{gathered}
\frac{d x}{d t}=\alpha x(t)-\beta x(t) y(t)-\gamma x(t)^{2}, \\
\frac{d y}{d t}=-\rho y(t)+\sigma x(t) y(t)-\nu y(t)^{2},
\end{gathered}
$$

where $\alpha, \beta, \gamma, \rho, \sigma, \nu$ are all positive parameters. $x(t)$ represent the prey population and $y(t)$ represent the predator population. $\alpha$ is the growth rate of prey population, $\beta$ attack rate of the prey by predator, $\gamma$ is the strength of intraspecies of competition among the prey population, $\rho$ is death rate of predator population, $\sigma$ represent the conversion rate from prey to predator and $\nu$ is the strength of competition among the predator population.

Qualitative investigation of (1) and (2) reveals the following equalibrium points

$$
E_{0}(0,0)
$$

and

$$
E_{1}\left(\frac{\alpha}{\gamma}, 0\right)
$$

and

$$
E_{2}\left(\frac{\beta \rho+\alpha \nu}{\sigma \beta+\gamma \nu}, \frac{\alpha \sigma-\gamma \rho}{\sigma \beta+\gamma \nu}\right)
$$


Let us set

$$
\begin{aligned}
& f(x, y)=\alpha x(t)-\beta x(t) y(t)-\gamma x(t)^{2}, \\
& g(x, y)=-\rho y(t)+\sigma x(t) y(t)-\nu y(t)^{2} .
\end{aligned}
$$

Jacobian matrix is

$$
J_{\left(x^{*}, y^{*}\right)}=\left[\begin{array}{cc}
f_{x} & f_{y} \\
g_{x} & g_{y}
\end{array}\right]
$$

Let us consider $E_{0}(0,0)$ :

$$
J_{(0,0)}=\left[\begin{array}{cc}
\alpha & 0 \\
0 & -\rho
\end{array}\right]
$$

Since the eigevalues $\lambda_{1}=\alpha$ ans $\lambda_{2}=-\rho$ have opposite signs then $E_{0}(0,0)$ is a saddle node.

For $E_{1}\left(\frac{\alpha}{\gamma}, 0\right)$ :

$$
J_{\left(\frac{\alpha}{\gamma}, 0\right)}=\left[\begin{array}{cc}
-\alpha & \frac{-\alpha \beta}{\gamma} \\
0 & -\rho+\frac{\alpha \sigma}{\gamma}
\end{array}\right]
$$

Eigevalues $\lambda_{1}=-\alpha$ and $\lambda_{2}=-\rho+\frac{\alpha \sigma}{\gamma}, E_{1}\left(\frac{\alpha}{\gamma}, 0\right)$ is a locally asymtotically stable provided $\alpha \sigma<\rho \gamma$.

For $E_{2}\left(\frac{\beta \rho+\alpha \nu}{\sigma \beta+\gamma \nu}, \frac{\alpha \sigma-\gamma \rho}{\sigma \beta+\gamma \nu}\right)$ :

$$
J_{\left(x^{*}, y^{*}\right)}=\left[\begin{array}{cc}
-\gamma x^{*} & -\beta x^{*} \\
\sigma y^{*} & -\sigma y^{*}
\end{array}\right]
$$

Considering Trace $(J)=-\gamma x^{*}-\sigma y^{*}<0$ and

$$
\operatorname{det}\left(J=\sigma \gamma x^{*} y^{*}+\sigma \beta x^{*} y^{*}=x^{*} y^{*}(\sigma \gamma+\sigma \beta)>0\right.
$$

Characteristics polynomial will be $\lambda^{2}-\operatorname{Trace}(J) \lambda+\operatorname{det}(J)=0$ and the eigenvalues $\lambda_{1}$ and $\lambda_{2}$ will strictly have negative real parts provided $\alpha \sigma<\rho \gamma$.

Thus $E_{2}\left(\frac{\beta \rho+\alpha \nu}{\sigma \beta+\gamma \nu}, \frac{\alpha \sigma-\gamma \rho}{\sigma \beta+\gamma \nu}\right)$ is a locally asymtotically stable.

\section{Computational Method}

Re-writting both equations (1) and (2) in an integral form

$$
x(t)=x(0)+\alpha \int_{0}^{t} x d t-\beta \int_{0}^{t} x y d t-\gamma \int_{0}^{t} x^{2} d t,
$$




$$
y(t)=y(0)-\rho \int_{0}^{t} y d t+\sigma \int_{0}^{t} x y d t-\nu \int_{0}^{t} y^{2} d t .
$$

Applying the Adomian decomposition algorithm to (6) and (7), we receive

$$
x=\sum_{n=0}^{\infty} x_{n}, \quad y=\sum_{n=0}^{\infty} y_{n} .
$$

The non-linear terms are approximated as

$$
\begin{gathered}
x^{2}=\sum_{n=0}^{\infty} G_{n}\left(x_{0}, \ldots x_{n}\right), \quad y^{2}=\sum_{n=0}^{\infty} H_{n}\left(y_{0}, \ldots . y_{n}\right), \\
x y=\sum_{n=0}^{\infty} K_{n}\left(x_{0}, \ldots, x_{n}, y_{0}, \ldots . y_{n}\right),
\end{gathered}
$$

where

$$
\begin{aligned}
& G_{n}=\frac{1}{n !} \frac{d^{n}}{d \mu^{n}}\left[\sum_{n=0}^{\infty}\left(\mu^{n} x_{n}\right)^{2}\right]_{\mu=0}, \\
& H_{n}=\frac{1}{n !} \frac{d^{n}}{d \mu^{n}}\left[\sum_{n=0}^{\infty}\left(\mu^{n} y_{n}\right)^{2}\right]_{\mu=0},
\end{aligned}
$$

and

$$
K_{n}=\frac{1}{n !} \frac{d^{n}}{d \mu^{n}}\left[\sum_{n=0}^{\infty} \mu^{n} x_{n} \sum_{n=0}^{\infty} \mu^{n} y_{n}\right]_{\mu=0} .
$$

The non-linear terms $G_{n}, H_{n}$ and $K_{n}$ are the Adomian polynomials. Substituting (8)- (13) into (6) and (7)

$$
\begin{aligned}
& \sum_{n=0}^{\infty} x_{n}=x(0)+\alpha \int_{0}^{t} \sum_{0}^{\infty} x_{n} d t-\beta \int_{0}^{t} \sum_{0}^{\infty} K_{n} d t-\gamma \int_{0}^{t} \sum_{0}^{\infty} G_{n} d t, \\
& \sum_{n=0}^{\infty} y_{n}=y(0)-\rho \int_{0}^{t} \sum_{0}^{\infty} y_{n} d t+\sigma \int_{0}^{t} \sum_{0}^{\infty} K_{n} d t-\nu \int_{0}^{t} \sum_{0}^{\infty} H_{n} d t .
\end{aligned}
$$

Equations (15) and (16) can be written as recussive formula

$$
x_{n+1}=\alpha \int_{0}^{t} x_{n} d t-\beta \int_{0}^{t} K_{n} d t-\gamma \int_{0}^{t} G_{n} d t,
$$




$$
y_{n+1}=-\rho \int_{0}^{t} y_{n} d t+\sigma \int_{0}^{t} K_{n} d t-\nu \int_{0}^{t} H_{n} d t,
$$

where $x_{0}=x(0)$ and $y_{0}=y(0)$.

The Adomian polynomials for (11), (12) and (13) are:

$$
\begin{aligned}
& G_{0}=x_{0}^{2}, \\
& G_{1}=2 x_{0} x_{1}, \\
& G_{2}=x_{1}^{2}+2 x_{0} x_{2}, \\
& G_{3}=2 x_{1} x_{2}+2 x_{0} x_{3}, \\
& G_{4}=x_{2}^{2}+x_{1} x_{3}+2 x_{0} x_{4}, \\
& G_{5}=2 x_{2} x_{3}+2 x_{1} x_{4}+2 x_{0} x_{5}, \\
& \vdots \\
& H_{0}=y_{0}^{2}, \\
& H_{1}=2 y_{0} y_{1}, \\
& H_{2}=y_{1}^{2}+2 y_{0} y_{2}, \\
& H_{3}=2 y_{1} y_{2}+2 y_{0} y_{3}, \\
& H_{4}=y_{2}^{2}+y_{1} y_{3}+2 y_{0} y_{4}, \\
& H_{5}=2 y_{2} y_{3}+2 y_{1} y_{4}+2 y_{0} y_{5},
\end{aligned}
$$

and

$$
\begin{aligned}
& K_{0}=x_{0} y_{0} \\
& K_{1}=x_{1} y_{0}+x_{0} y_{1}, \\
& K_{2}=x_{2} y_{0}+x_{1} y_{1}+x_{0} y_{2}, \\
& K_{3}=x_{3} y_{0}+x_{2} y_{1}+x_{1} y_{2}+x_{0} y_{3}, \\
& K_{4}=x_{4} y_{0}+x_{3} y_{1}+x_{2} y_{2}+x_{1} y_{3}+x_{0} y_{4}, \\
& K_{5}=x_{5} y_{0}+x_{4} y_{1}+x_{3} y_{2}+x_{2} y_{3}+x_{1} y_{4}+x_{0} y_{5},
\end{aligned}
$$

respectively. 


\section{Numerical Results}

In this section we do numerical evaluation of (1) and (2) using the Adomian decomposition method and using all the positive parameters. The results are:

\begin{tabular}{ccccccccc}
\hline Cases & $x_{0}$ & $y_{0}$ & $\alpha$ & $\beta$ & $\gamma$ & $\rho$ & $\sigma$ & $\nu$ \\
\hline case 1 & 0.4 & 0.5 & 0.001 & 0.3 & 0.1 & 0.1 & 0.1 & 0.1 \\
case 2 & 0.4 & 0.5 & 0.1 & 0.6 & 0.1 & 0.4 & 0.1 & 0.1 \\
case 3 & 0.4 & 0.5 & 0.9 & 0.4 & 0.1 & 0.5 & 0.4 & 0.1 \\
case 4 & 0.4 & 0.5 & 0.4 & 0.1 & 0.01 & 0.1 & 0.4 & 0.01 \\
\hline
\end{tabular}

$$
\begin{aligned}
x(t)= & .5-0.0845 t+0.0119527 t^{2}-0.00144726 t^{3}+0.000159027 t^{4} \\
& -0.0000163988 t^{5}+1.62885 \times 10^{-6} t^{6}, \\
y(t)= & 0.4-0.036 t+0.00065 t^{2}+0.000189403 t^{3}-0.0000315888 t^{4} \\
& +3.23513 \times 10^{-5} t^{5}-2.50305 \times 10^{-7} t^{6}, \\
x(t)= & 0.5-0.095 t+0.0348 t^{2}-0.00921283 t^{3}+0.00231238 t^{4} \\
& -0.000549775 t^{5}+0.000124719 t^{6} \\
y(t)= & 0.4-0.156 t+0.03164 t^{2}-0.00438827 t^{3}+0.000415537 t^{4} \\
& -5.53856 \times 10^{-6} t^{5}-0.0000105528 t^{6} \\
x(t)= & 0.5+0.345 t+0.124 t^{2}+0.0251792 t^{3}+0.0020838 t^{4} \\
& -0.000538282 t^{5}-0.000244219 t^{6} \\
y(t)= & 0.4-0.136 t+0.05344 t^{2}-0.00702827 t^{3}+0.00219552 t^{4} \\
& -0.000133333 t^{5}+0.0000797715 t^{6} \\
x(t)= & 0.5+0.1775 t+0.0301025 t^{2}+0.00291363 t^{3}+0.0000909901 t^{4} \\
& -0.0000258229 t^{5}-6.76607 \times 10^{-6} t^{6}, \\
y(t)= & 0.4+0.0384 t+0.0159664 t^{2}+0.00299899 t^{3}+0.000581454 t^{4} \\
& +0.000102627 t^{5}+0.0000168842 t^{6} .
\end{aligned}
$$




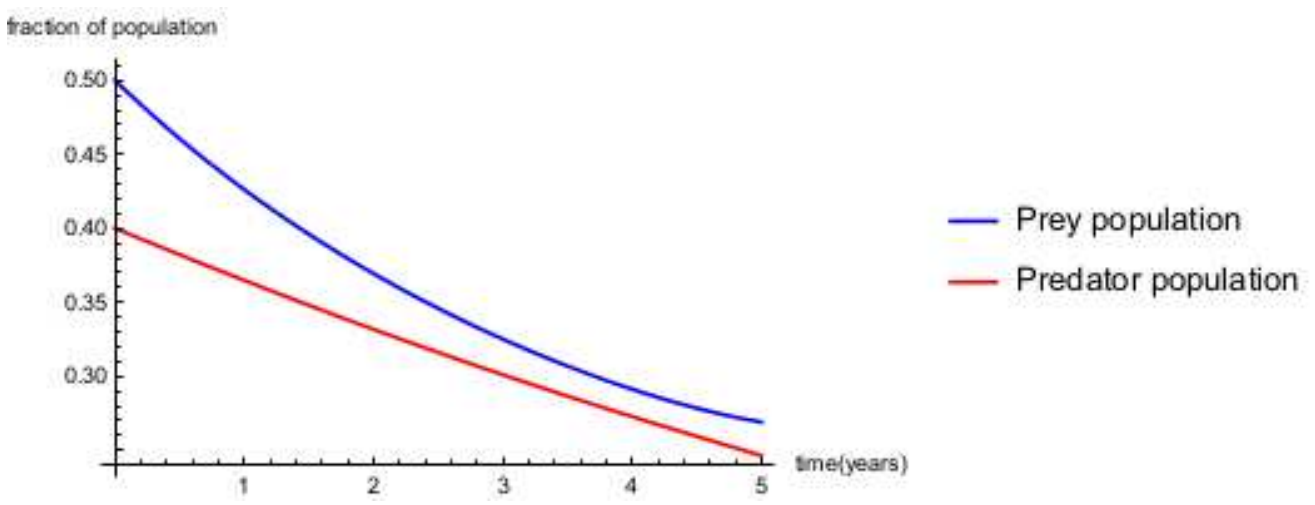

Figure 1: Generated from Case1

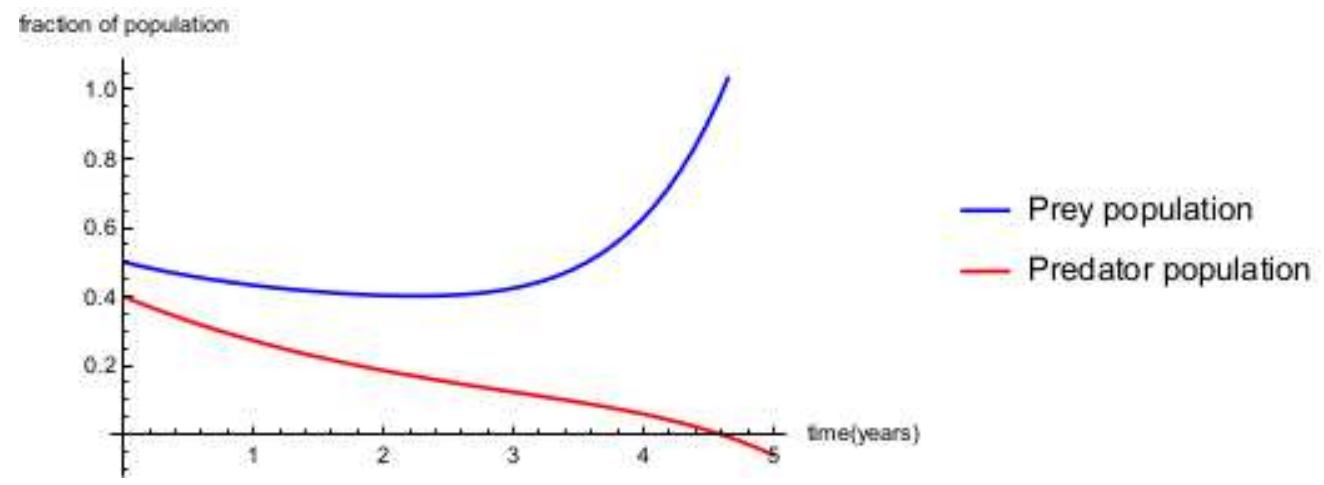

Figure 2: Generated from Case2

\section{Discussion}

The case 1 solutions indicate behaviour of the systems at $E_{0}$ where both prey and predator will die out of the system(extinction). Case 2 respresent $E_{1}$ where the prey will remain in the system after long period of time(predator extinction). Case 3 and Case 4 represent stable co-existence $E_{2}$ where both prey and predator will remain in they system considering the present of intraspecies competition. Adomian decomposition method offers alternative ways of approximating any given initial-valued system of non-linear diffferential equations. The method is easy to compute does not involve and complex computations. 


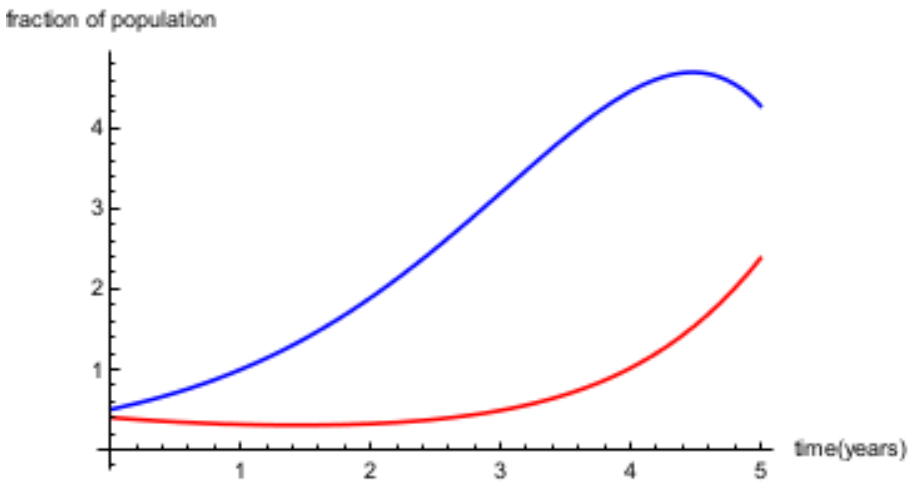

- Prey population

- Predator population

Figure 3: Generated from Case3

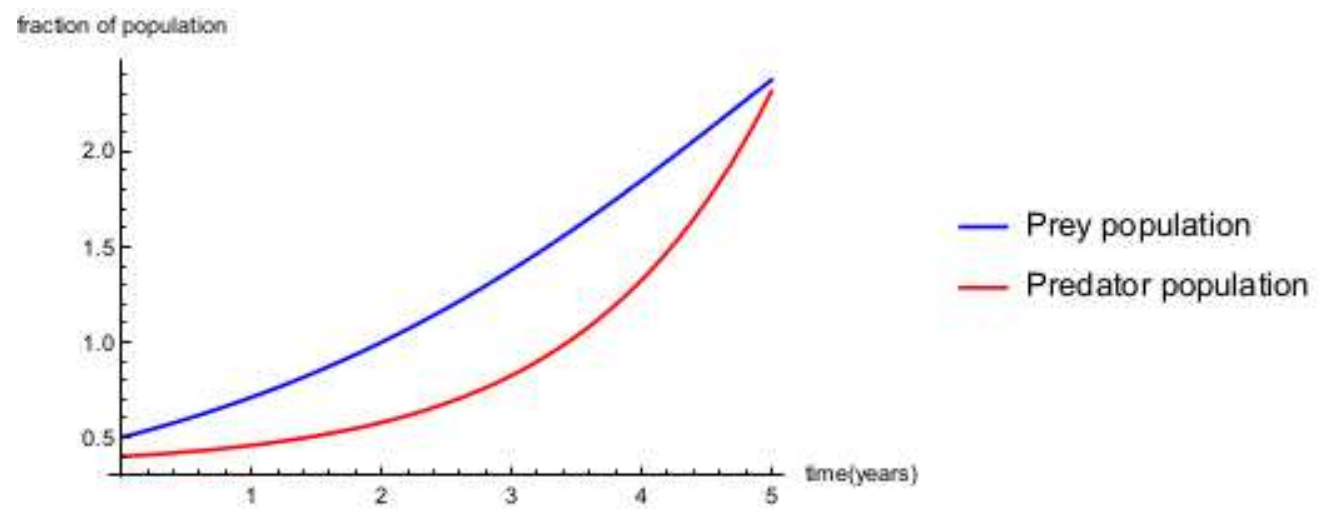

Figure 4: Generated from Case4

\section{Acknowledgments}

The author like to thank the Tshwane University of Technology for their financial support.

\section{References}

[1] G. Adomian, Solving Frontier Problems of Physics, Kluwer, Boston, 1994.

[2] G. Adomian, Nonlinear Stochastic Operator Equations Frontier, Academic Press, San Diego, 1986.

[3] G. Adomian, A review of the decomposition method and some recent results for nonlinear equation, Math.Comput. Modelling, 7 (1992), 17-43. 
[4] G. Adomian, R.Rach, Equality of partial solutions in the decomposition method for linear or nonlinear partial defferential equations, Comput. Math. Appl., 12 (1990), 9-12.

[5] A Bazykin, Nonlinear Dynamics of Interacting Populations, World Scientific Pub Co Inc, 1998.

[6] J. Biazar, A, Montazeri, A computational method for solution of the prey and predator problems, Comput. Math. Appl., 163 (2005), 841 -847.

[7] J.Y. Edwards, J.A. Roberts, N.J. Ford, A comparision of Adomian's decomposition method and Runge-Kutta methods for approximate solution of some predator-prey model equations, Manchester Centre of Computational Mathematics (1997), 1-17.

[8] G. Adomian, R.Rach, Equality of partial solutions in the decomposition method for linear or nonlinear partial defferential equations, Comput. Math. Appl., 12 (1990), 9-12.

[9] O.D Makinde, Solving ratio-dependant prey-predator system with constant effort harvesting using Adomian decomposition method, Comput. Math. Appl., 186 (2007), 17-22.

[10] R. N. Shalan, R.K Naji, The dynamics of Holling Type IV prey predator with intraspecific competition, Iraqi Journal of Science, 54 (2013), 386-396. 
